\title{
A Case of Colonic Hypoganglionosis Complicated with Colonic Ulcers
}

\author{
Kyu-Man Cho', Sung-Uk Lim', Seon-Young Park', Kyung-Hwa Lee², Jae-Kyun Ju³, Jong-Sun Rew' \\ Departments of ${ }^{1}$ Internal Medicine, ${ }^{2}$ Pathology, and ${ }^{3}$ Surgery, Chonnam National University Medical School, Gwangju, Korea
}

\begin{abstract}
Hypoganglionosis is a rare form of intestinal neuronal malformation, which is characterized by reduced number and size of ganglion cells of parasympathetic nerves in the intestinal wall. Pathophysiology is not well known, however intestinal ischemia, inflammation, autoimmune process or neurotoxin may play a role. Here, we report the case of a 56-year-old man with colonic pseudoobstruction and ulcerations in marked dilatedcolon above transitional zone who was later diagnosed with colonic hypoganglionosis.

Keywords: Ganglion; Pseudo-obstruction; Ulcer
\end{abstract}

\section{INTRODUCTION}

Hypoganglionosis is a hypogenetic type of intestinal innervation disorder, characterized by sparse and small myenteric ganglia in the region of colon and rectum [1]. Hypoganglionosis occurs in two sub-classification according to onset time: congenital hypoganglionosis, and acquired hypoganglionosis, in which cause of ganglia degeneration is not well known, but intestinal ischemia, inflammation, autoimmune process or neurotoxin may play a role [2]. Patient with acquired hypoganglionosis may present with constipation, abdominal pain, abdominal distension, or intestinal pseudo-obstruction without obstructive lesions. Acquired hypoganglionosis has good prognosis after performing an appropriate resection of the affected bowel [2]. Here, we report the case of a 56 -year-old man with colonic pseudoobstruction and ulcerations in marked dilated colon above transitional zone who was later diagnosed with colonic hypoganglionosis.

\section{CASE REPORT}

A 56-year-old male patient was admitted to our emergency room with complaints of progressively increasing abdominal distension and pain. He suffered from recurrent constipation and abdominal distention for 5 years, which had been improved through use of laxatives or enema. He had been unable to pass stool or gas passage for three day with no response to laxatives or prokinetics. Physical examination revealed abdominal distension with hypoactive bowel sound, and he complained of tenderness at left lower quadrant without rebound tenderness. A digital rectal examination was normal. Routine laboratory evaluation was normal, including complete blood cell count, urinalysis, biochemical tests, and thyroid test. Bowel distension in especially transverse colon was seen in the simple abdominal X-ray. Abdominal computed tomography (CT) scan showed diffuse dilatation from ascending colon to descending colon with a transition zone in the sigmoidodescending (SD) junction (Fig. 1). Transition zone ratio (the transverse diameter ratio of the most dilatated colonic segment proximal to the transition zone to the narrowed colonic segment distal to the transition zone) was 8.75. Colonoscopy showed highly narrowed segment with need of forced pushing in the SD junction, and dilated loop with no haustration in the proximal colon. Also, there were well demarcated geographic ulcers with longitudinal array from splenic flexure to distal transverse colon (Fig. 2). In histopathology, we can find out pathologic lesion present 7 to $8 \mathrm{~cm}$ away from the distal resection margin, corresponding to SD junction. Biopsy specimens from mucosa in the transition zone and ulcer in the proximal colon showed pathologically nonspecific findings. We did not perform any constipation study such as colon transit time, fecal anography 

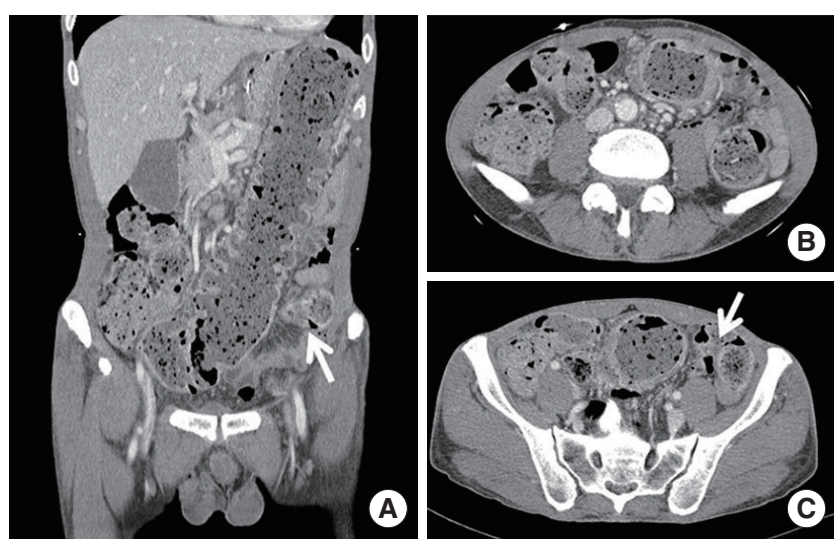

Fig. 1. Abdominal computed tomography scan. (A, B) It shows diffuse dilatation and wall thickening of transverse colon. (C) Focal narrowed transitional zone (white arrow) was observed at the rectosigmoid junction.

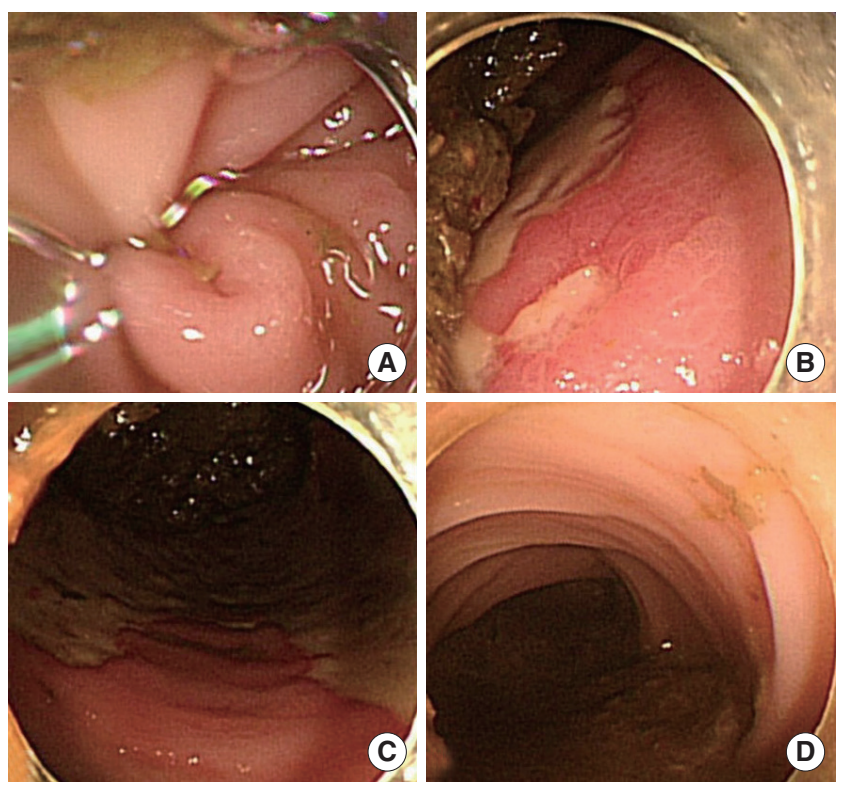

Fig. 2. Colonoscopic findings. (A) Focal strictured segment with normal mucosa are observed at the rectosigmoid junction. (B, C) Several geographic and longitudinal ulcers are observed on the distended transverse colon. (D) Proximal dilated colon with decreased haustration are shown.

or anorectal manometry, because we thought that it was dangerous to perform such exams due to patient's continuous left lower quadrant pain and tenderness. Seven days after admission, we decide to perform emergency operation due to no response to laxatives or enema and possibility of decompensation of proximal colon. $\mathrm{He}$ underwent subtotal colectomy with end ileostomy. Gross specimen showed the $3 \mathrm{~cm}$ long transition zone in distal descending colon, diffuse dilatation from proximal left colon to ascending colon, and multiple longitudinal ulcerative lesions in transverse colon, which were compatible to CT and colonoscopic findings (Fig. 3). Histo-

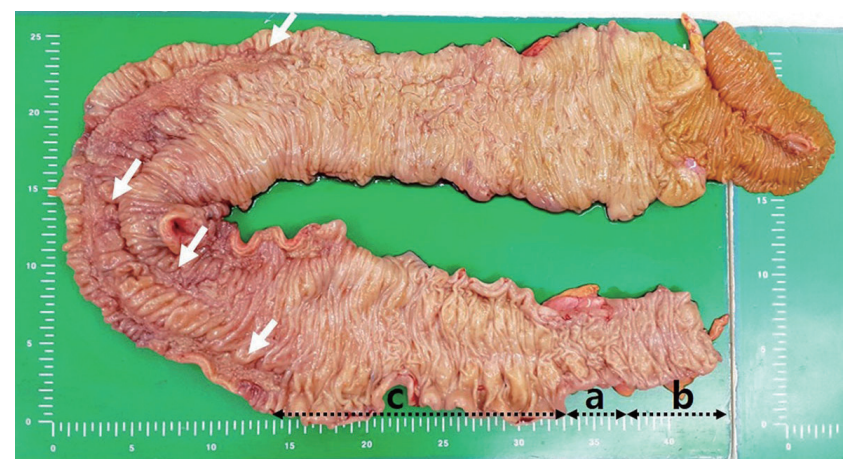

Fig. 3. Postoperative gross finding. It shows $3 \mathrm{~cm}$ length of focal narrowed segment (a) away from distal resected margin, proximal gradullay dilated colon (c) and longitudinal ulcers (white arrows). Distal segment (b) to transitional zone (a) shows normal morphology.
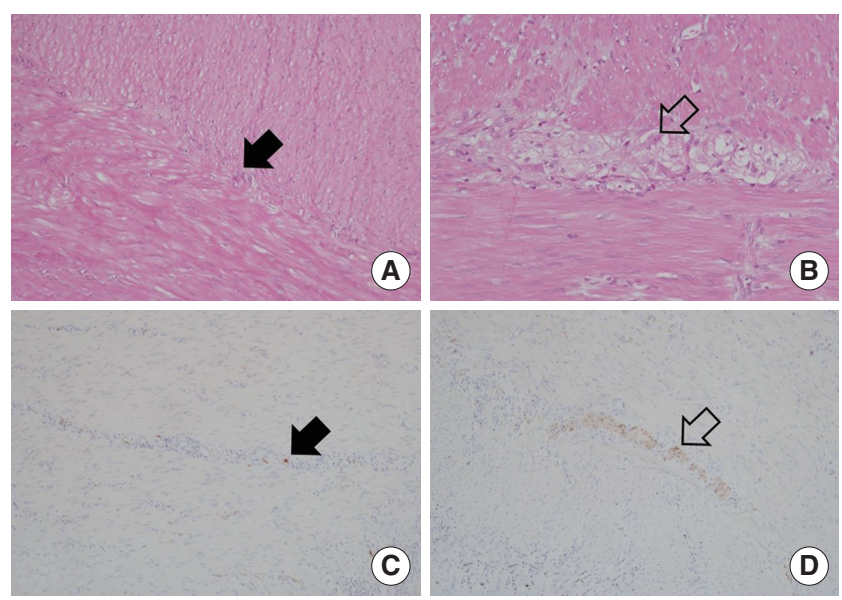

Fig. 4. H\&E stain and immunohistochemical stain using c-kit and B-cell lymphoma 2 (Bcl-2) in the myenteric plexus. It shows marked decreased ganglion cells, the reduction of intersitial cells of Cajal (black arrows) and immature ganglion cells (white arrows) in the myenteric plexus of transition zone. (A) H\&E stain, $\times$ 100. (B) H\&E stain, $\times 200$. (C) C-kit stain, $\times 100$. (D) Bcl-2 stain, $\times 100$.

logic examination of the transition showed marked decreased number of mature myenteric ganglion cells and decreased size of ganglions in themyenteric plexus with hypertrophied circular muscle layer and relatively atrophied outer longitudinal muscle layer (calculated number of ganglion cells per $1 \mathrm{~cm}$ in $\mathrm{TZ}$ and no TZ). Immunohistochemical stain for c-kit, B-cell lymphoma 2 (Bcl-2) were markedly decreased in the myenteric plexus (acetylcholinesterase $[\mathrm{AChE}]$, nicotinamide adenine dinucleotide phosphate [NADPH]-diaphorase) (Fig. 4). Microscopic finding of proximal colon showed normal number and shape of ganglion cells. He has been followed-up with improvement in his bowel habit (once a day) and his body weight, and quality of life after surgery. 


\section{DISCUSSION}

Hypoganglionosis could be classified into two distinct subgroups; focal type (type I) and diffuse type (type II), with or without a focally narrowed segment (so called transition zone), which have a few ganglion cells between inner circular and outer longitudinal intestinal muscle [3]. Our present case was relevant to focal type or type I hypoganglionosis, which had a focally narrowed segment of transition zone in SD junction on abdomen CT and colonoscopy. Hypoganglionosis in adult could be also divided into congenital and acquired hypoganglionosis according to onset time. The clinical characteristics as well as the histological findings are apparently different between congenital and acquired hypoganglionosis.

Pathogenesis of gangliadegeneration in acquired hypoganglionosishas not yet been well-known, however intestinal ischemia, inflammation, autoimmune process or neurotoxin may play a role for chronic intestinal pseudo-obstruction [4].

CT scan is a use imaging tool providing the opportunity to not only view the dilated colon and transition zones but also to definitively exclude other diseases which can also cause chronic constipation in adults, such as colon cancer [5]. In this case, CT scan showed diffuse dilatation from ascending colon to descending colon with a transition zone in the SD junction. A role of colonoscopy was not well known in diagnosis of hypoganglionosis. However, specific findings in colonoscopy, such as forced pushing of scope and tripping sense on transitional zone without any mucosal lesion and proximal dilated colon loop with decreased haustal markings like in this case could be suspected to hypoganglionosis. Additionally there were longitudinal geographic ulcerative lesions in proximal dilated colon in the case. Colonic ulceration could be shown in various disease: non-steroidal antiinflammatory drugs colopathy, ischemia, rectal trauma, solitary rectal ulcer syndrome, stercoral ulcers, infectious colitis, Behcet's syndrome, collagen/vascular disease, and neoplasia [6]. However, in this case, there was no evidence of autoimmune disease or infectious diseases. Concretely, we did additional immunohistochemical stains, and finally we revealed results of herpes simplex virus (-), cytomegalovirus (-), methenamine-silver $(-)$, periodic acid-Schiff stain (-), and acid-fast bacilli stain (-). Multiple geographic ulcers in upstream dilated colon could occur by ischemic damage from high pressure. Increasing intraluminal pressure could impair capillary perfusion. In sigmoid volvulus, mechanical obstruction because of twisting of mesenteric vessels and thrombosis of mesosigmoid veins contribute to ischemia. Ischemic injury occurring in the mucosa earlier than in other colonic layers facilitates bacterial translocation and toxaemia. In the present case, colonic ischemia due to increased intraluminal pressure, bacterial translocation, and toxemia may contributed to decompensation of proximal colon. Therefore, we decided to perform urgent colectomy without delay based on colonoscopic findings.

In 1990, a consensus conference recommended the examination of at least three full-thickness bowel biopsies to assess characteristic feature of the disease: very low mucosal activity of AchE, significant deficiency of nerve cells in the myenteric plexus, and hypertrophic muscularis mucosae and circular muscle layers [7]. Though decreased ganglion cells could be checked in hematoxylin \& eosin stain, low or absent activity of AchE in immunohistochemical (IHC) stain is used to confirm the diagnosis. Also other commonly used additional markers include staining of the biopsy for lactate dehydrogenase, succinate dehydrogenase, NADPH-diaphorase, ckit, interstitial cells of Cajal or silver staining, and S-100 staining [1]. In our case, hypoganglionosis was confirmed by using IHC stain of c-kit which was found a reduced immunoreactivity for intestinal pacemaker activity in hypoganglionosis, and Bcl-2 which is useful marker of immature enteric ganglion cells in affected lesion [8].

Patients with acquired hypoganglionosis in adult usually underwent a colectomy of affected colon segment for diagnosis and treatment because it is very difficult to perform the full-thickness bowel biopsy through colonoscopy unlike congenital hypoganglionosis in children patients. According to the literature, acquired hypoganglionosis could show a favorable prognosis unlike congenital hypoganglionosis if proper surgery is performed. However, occasionally additional resection could be required because of unsatisfactory clinical results and the recurrence of chronic constipation through a inadequate resection. Therefore, securing a sufficient distal margin and applying multiple full-thickness biopsies around the lesion with staining AChE during operation could be need. Gladman et al. [9] recommended that colectomy appears to be the optimum procedure in patients with a nondilated rectum, restorative proctocolectomy the most suitable in those with dilatation of the colon and rectum, and vertical reduction rectoplasty in those patients with dilatation confined to the rectum. After our patient underwent a subtotal colectomy with ileostomy, had a good bowel movement without recurrence during follow-up.

Acquired hypogangionosis in adult is a rare disease with acute or chronic colonic pseudo-obstruction. We report a case of acquired colonic hypoganglionosis with decompensation of proximal colon 
in adult. If possible, endoscopic examination of dilated proximal colon through transition zone with a gentle manipulation could provide the meaningful evidence for the prognosis and decision making of treatment strategy.

\section{REFERENCES}

1. Dingemann J, Puri P. Isolated hypoganglionosis: systematic review of a rare intestinal innervation defect. Pediatr Surg Int 2010;26:1111-5.

2. Taguchi T, Masumoto K, Ieiri S, Nakatsuji T, Akiyoshi J. New classification of hypoganglionosis: congenital and acquired hypoganglionosis. J Pediatr Surg 2006;41:2046-51.

3. Do MY, Myung SJ, Park HJ, Chung JW, Kim IW, Lee SM, et al. Novel classification and pathogenetic analysis of hypoganglionosis and adult-onset Hirschsprung's disease. Dig Dis Sci 2011;56:1818-27.

4. Wedel T, Roblick U, Gleiss J, Ott V, Eggers R, Kuhnel W, et al. Disorders of intestinal innervation as a possible cause for chronic constipation. Zentralbl Chir 1999;124:796-803.

5. Antonucci A, Fronzoni L, Cogliandro L, Cogliandro RF, Caputo C, De Giorgio R, et al. Chronic intestinal pseudo-obstruction. World J Gastroenterol 2008;14:2953-61.

6. Nagar AB. Isolated colonic ulcers: diagnosis and management. Curr Gastroenterol Rep 2007;9:422-8.

7. Borchard F, Meier-Ruge W, Wiebecke B, Briner J, Muntefering H, Fodisch HF, et al. Disorders of the innervation of the large intestine: classification and diagnosis: results of a consensus conference of the Society of Gastroenteropathology 1 December 1990 in Frankfurt/Main. Pathologe 1991;12: 171-4.

8. Rolle U, Yoneda A, Solari V, Nemeth L, Puri P. Abnormalities of C-Kit-positive cellular network in isolated hypoganglionosis. J Pediatr Surg 2002;37: 709-14.

9. Gladman MA, Scott SM, Lunniss PJ, Williams NS. Systematic review of surgical options for idiopathic megarectum and megacolon. Ann Surg 2005;241:562-74. 